\title{
Fast Inverter Loss and Temperature Simulation and Silicon Carbide Device Evaluation for Hybrid Electric Vehicle Drives
}

\author{
Angus Bryant Non-member (School of Engineering, University of Warwick, \\ Coventry, CV4 7AL, UK) \\ Graham Roberts Non-member (School of Engineering, University of Warwick, \\ Coventry, CV4 7AL, UK) \\ Alan Walker Non-member (Formerly School of Engineering, University of Warwick, UK; \\ now AVL Powertrain UK, Sylvan Way, Basildon, SS15 6TW, UK) \\ Philip Mawby Non-member (School of Engineering, University of Warwick, \\ Coventry, CV4 7AL, UK) \\ Takashi Ueta Non-member (Toyota Motor Corporation, 543, Kirigahora, \\ Nishihirose-Cho, Toyota, Aichi 470-0309, Japan) \\ Tosifumi Nisijima Non-member (Toyota Motor Corporation, 543, Kirigahora, \\ Nishihirose-Cho, Toyota, Aichi 470-0309, Japan) \\ Kimimori Hamada Member (Toyota Motor Corporation, 543, Kirigahora, \\ Nishihirose-Cho, Toyota, Aichi 470-0309, Japan)
}

Keywords: silicon carbide, compact modeling, power semiconductor devices, hybrid vehicle, driving cycle

This paper presents an integrated framework for modelling inverter performance and evaluating power devices in hybrid electric vehicle drives. New material devices, e.g. silicon carbide ( $\mathrm{SiC}$ ), also offer potential improvements over existing silicon ( $\mathrm{Si}$ ) devices, e.g. reduced losses, increased operating temperatures and smaller size.

Compact physics-based device models offers greatly improved accuracy in the simulation of inverter losses; however these require simulation times of up to a few seconds for every switching cycle. This work decouples the device and inverter simulation to maintain high accuracy of power losses and devices temperatures, and achieve faster than real time inverter simulation.

The key to the inverter simulation is the look-up table of device losses. During simulation, the inverter model simply accesses a look-up table of device losses, given as a function of the load current, duty ratio and device temperature. The table is pre-calculated using accurate compact device models. The IGBT and PIN diode models use the Fourier-based solution to calculate the stored charge, and therefore predict conduction and switching losses. A SiC Schottky diode model was also developed. Both the device models and inverter simulation are implemented in MATLAB/Simulink, giving full integration.

Look-up tables were then generated for two combinations: the Si IGBT with Si PIN diode, and Si IGBT with SiC Schottky diode. Since the switching frequency is relatively low at $5 \mathrm{kHz}$, and the onstate losses of the SiC Schottky diode are higher than that of the $\mathrm{Si}$ PIN diode, the losses are greater than those for the Si PIN diode. However, at a switching frequency of $25 \mathrm{kHz}$, the losses are similar, indicating the improved performance of SiC Schottky diode at high switching frequencies.

A candidate D-segment full parallel hybrid vehicle was used in this paper to provide a suitable load cycle for inverter simulation. The driving cycles used are the combined Artemis cycle and the NEDC. Temperature profiles were generated for both pairs of devices and for both driving cycles. The temperatures depend on the vehicle torque, with peaks in temperature corresponding to the vehicle acceleration and motor torque. The temperatures show greater peaks and more severe cycling during the urban section, which would adversely impact the device reliability. The maximum temperature rise occurs during sharp braking. There is little difference between the device temperatures simulated using the Si PIN diode and those using the SiC Schottky diode, because of the strong dependence on the on-state losses at the switching frequency used. The speed-up factors of inverter simulation were at least 3.3 times faster than real time.

The SiC Schottky diode shown here achieves a similar performance to an already well-optimized Si PIN diode, at the expense of using a diode chip area more than double that of the Si PIN diode. The potential drawback of high on-state losses of SiC Schottky diodes has not been widely discussed, since it is assumed that such diodes operate at high switching frequencies. The ability of $\mathrm{SiC}$ to operate at higher temperatures would not restrict the diode temperature to the same extent, allowing smaller diode chip sizes to be used, but this could increase the temperature of an adjacent IGBT and affect the packaging reliability through thermal cycling. 


\section{Fast Inverter Loss and Temperature Simulation and Silicon Carbide Device Evaluation for Hybrid Electric Vehicle Drives}

$\begin{array}{ll}\text { Angus Bryant }^{*} & \text { Non-member } \\ \text { Graham Roberts }^{*} & \text { Non-member } \\ \text { Alan Walker } & \text { Non-member } \\ \text { Philip Mawby } & \text { Non-member } \\ \text { Takashi Ueta** } & \text { Non-member } \\ \text { Tosifumi Nisijima }^{* * *} & \text { Non-member } \\ \text { Kimimori Hamada }^{* * *} & \text { Member }\end{array}$

This paper presents an integrated framework for modelling inverter performance and evaluating power devices in hybrid electric vehicle drives. Based in MATLAB/Simulink, it uses a novel method of decoupling the device and inverter simulation to maintain high accuracy of power losses and devices temperatures, and achieve faster than real time inverter simulation. An illustration is given for a full hybrid vehicle for different driving cycles. Device models are included for silicon carbide Schottky diodes as well as silicon IGBTs and PIN diodes. Evaluation of the new material devices is performed, to estimate the potential performance gains achievable. The simulation framework offers the potential to rapidly improve the inverter and powertrain design process, and to evaluate device selection quickly.

Keywords: silicon carbide, compact modeling, power semiconductor devices, hybrid vehicle, driving cycle

\section{Introduction}

A major challenge in the design of a hybrid electric vehicle powertrain is the optimization of the motor drive inverter. The inverter transfers power between the battery and electric motor. The load on the inverter depends on the driving cycle and the powertrain configuration. This in turn sets the inverter rating, from which the power devices can be chosen and the heatsink designed. There exists a fundamental tradeoff between device performance, heatsink size and reliability, critically depending on the device temperature throughout vehicle operation. Reducing the inverter size (including the heatsink) is necessary in order to minimize the extra space occupied by the hybrid powertrain. New material devices, e.g. silicon carbide ( $\mathrm{SiC}$ ), also offer potential improvements over existing silicon ( $\mathrm{Si}$ ) devices, in terms of reduced losses, increased operating temperatures and smaller size.

Traditionally, inverter modelling has been carried out using circuit simulators, with either ideal switches or very basic device models representing the switching devices. While

\footnotetext{
Based on "Fast Inverter Loss Simulation and Silicon Carbide Device Evaluation for Hybrid Electric Vehicle Drives" by A.T. Bryant, G.J. Roberts, A. Walker and P.A. Mawby which appeared in the proceedings of the 2007 Power Conversion Conference-Nagoya, (C)2007 IEEE.

* School of Engineering, University of Warwick Coventry, CV4 7AL, UK

** Formerly School of Engineering, University of Warwick Coventry, CV4 7AL, United Kingdom; now AVL Powertrain UK, Sylvan Way, Basildon, SS15 6TW, UK

*** Toyota Motor Corporation

543, Kirigahora, Nishihirose-Cho, Toyota, Aichi 470-0309
}

the use of simple device switching models results in rapid inverter simulation, necessary for long driving cycles of many minutes, such models are unable to predict device losses and the resulting temperature rise with much accuracy. Therefore they cannot be used in inverter optimization. The use of compact physics-based device models offers greatly improved accuracy in the simulation of inverter losses ${ }^{(1)-(5)}$; however these require simulation times of up to a few seconds for every switching cycle. Clearly this approach cannot be used for more than tens of milliseconds of inverter operation, since the number of switching events would result in prohibitive simulation times.

In this paper a simulation framework is proposed which integrates hybrid powertrain modelling, inverter simulation and device modelling. The inverter simulation features a novel method of decoupling device switching behaviour from inverter and heatsink operation, so that accurate prediction of losses is maintained while allowing a fast simulation speed. The simulation framework is illustrated with a hybrid powertrain and a selection of driving cycles, and the effects of these on the device temperature is examined for different candidate devices.

\section{Modelling Framework}

The system model is illustrated in Fig. 1. The driving cycle data, namely the vehicle speed as a function of time, is used to generate the electric motor speed and torque using the powertrain model. A simple motor drive model then calculates the corresponding electrical conditions at the output of the inverter (rms voltage and current, power factor 


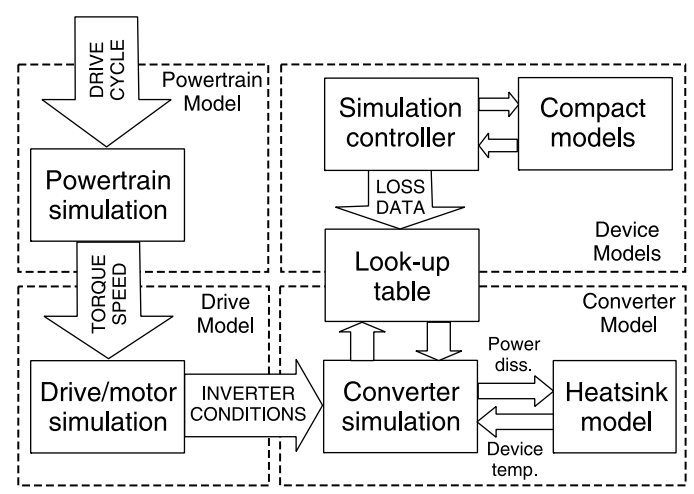

Fig. 1. Coupling between the parts of the system model. The powertrain, drive and device models are used independently of the converter model

and frequency). The inverter simulation then runs through the electrical conditions, generating the switching patterns and calculating the device losses and, in conjunction with a heatsink model, the device temperatures ${ }^{(6)}$.

The key to the inverter simulation is the look-up table of device losses. Rather than simulating device switching at every switching event, which is time-consuming, the inverter model simply accesses a look-up table of device losses ${ }^{(7)(8)}$. These losses are given as a function of the load current, duty ratio and device temperature, allowing rapid estimation of the device losses based on the switching conditions at the current time step. The look-up table is pre-calculated using accurate compact device models, with the advantage that simulation of device switching is only necessary once for any combination of switching conditions. The following sections explain the modelling stages in more detail.

2.1 Device Models The devices used in the inverter are IGBTs, with freewheel diodes to carry the inductive load current. The diodes simulated are either Si PIN diodes or SiC Schottky diodes.

2.1.1 Si PIN diode and IGBT Models The compact models used for the Si devices ${ }^{(9)}$, summarized in Fig. 2, account for the stored charge inside the IGBT and diode, which must be modelled to predict the on-state and switching losses accurately. They use Fourier series to solve the ambipolar diffusion equation (ADE) describing the excess stored charge $p(x, t)$ in the lightly-doped base region. The ADE is given by,

$$
D \frac{\partial^{2} p(x, t)}{\partial x^{2}}=\frac{p(x, t)}{\tau}+\frac{\partial p(x, t)}{\partial t}
$$

where $D$ is the ambipolar diffusivity and $\tau$ is the high-level lifetime. $p(x, t)$ can be expressed as a Fourier series in space $x$, with the coefficients $p_{k}$ varying in time $t$ :

$$
p(x, t)=\sum_{k=0}^{\infty} p_{k}(t) \cos \left(\frac{\pi k\left(x-x_{1}\right)}{x_{2}-x_{1}}\right) \ldots \ldots \ldots \ldots \ldots
$$

The models also account for device features such as local lifetime control in diodes ${ }^{(10)}$ and field-stop regions in IGBTs $^{(11)}$. The switching (commutation) cell and Si device models have previously been implemented in Simulink ${ }^{(6)(12)}$, which allows effective integration with MATLAB and generation of the look-up table of device losses. The models are described in detail in Refs. (9)-(12); readers are requested to refer to these papers for further details.

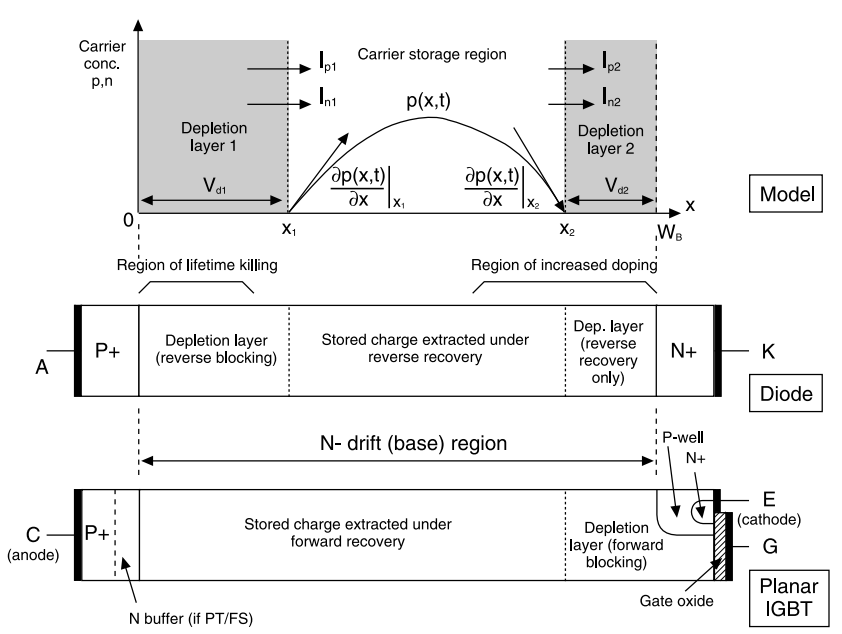

Fig. 2. Overview of compact Si IGBT and PIN diode models

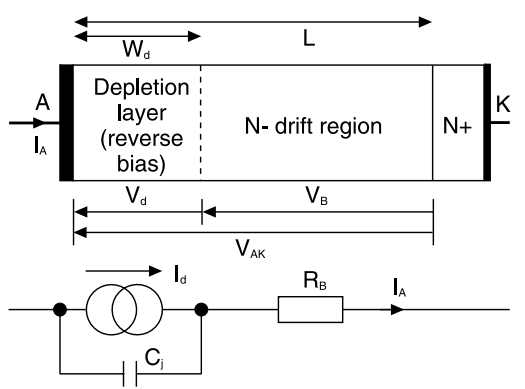

Fig. 3. Simplified structure of the Schottky diode for modelling

2.1.2 SiC Schottky Diode Model The SiC Schottky diode model is summarized in Fig. 3. It differs from the $\mathrm{Si}$ PIN diode model in that the Schottky diode is a unipolar device, and therefore it does not need to calculate the stored charge using the Fourier series solution. The Schottky diode drift region has a width $L$ and a doping concentration of $N_{D}$. The metal-semiconductor junction forms a depletion layer under reverse bias, which extends a width $W_{d}$ into the drift region. The remaining non-depleted drift region acts as a series resistance.

The forward current $I_{d}$ at the Schottky junction as a function of the junction voltage $V_{d}$ is given by,

$$
I_{d}=I_{0}\left(\exp \left(\frac{V_{d}}{n V_{T}}\right)-1\right),
$$

where $n$ is an ideality factor. $V_{T}=k T / q$ is the thermal voltage, where $k$ is the Boltzmann constant, $T$ is the absolute temperature and $q$ is the electronic charge. $I_{0}$ is the reverse saturation current ${ }^{(13)}$ :

$$
I_{0}=A A^{*} T^{2} \exp \left(-\frac{\phi_{B n}}{V_{T}}\right) .
$$

$A$ is the diode cross-sectional area, $\phi_{B n}$ is the barrier height and $A^{*}$ is the Richardson constant given by Ref. (13):

$$
A^{*}=\frac{4 \pi q m^{*} k^{2}}{h^{3}} \text {. }
$$

$m^{*}$ is the effective electron mass and $h$ is Planck's constant. The junction capacitance $C_{j}$ is given by: 


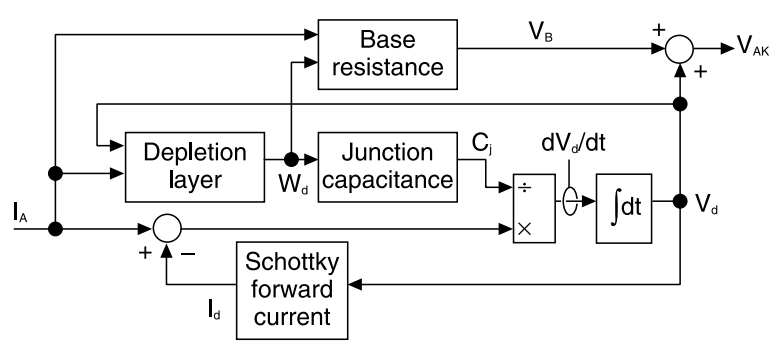

Fig. 4. Block diagram of the Schottky diode model

$$
C_{j}=\frac{\varepsilon A}{W_{d}}, \cdots
$$

where $\varepsilon$ is the dielectric constant for the semiconductor ( $\mathrm{Si}$ or $\mathrm{SiC}) . W_{d}$ is the depletion region width given by:

$$
W_{d}=\sqrt{\left(\frac{2 \varepsilon\left(V_{b i}-V_{d}-V_{T}\right)}{q N_{D}+\frac{\left|I_{A}\right|}{A v_{s a t}}}\right)}, \ldots \ldots \ldots \ldots \ldots \ldots
$$

where $V_{b i}$ is the built in junction potential, $N_{D}$ is the drift region doping concentration, $I_{A}$ is the total diode current and $v_{\text {sat }}$ is the electron saturation drift velocity.

The drift region resistance is given by Eq. (8). This takes into account that the depletion region extends into the drift region by $W_{d}$, reducing its effective length. The total drift region length is $L$.

$$
R_{B}=\frac{L-W_{d}}{q \mu_{n} N_{D} A}
$$

The temperature dependance of carrier mobility is given by Ref. (14):

$$
\mu_{n}=\frac{\mu_{n}(300)}{1+\left(\frac{N_{D}}{1.94 \times 10^{17}}\right)^{0.61}}\left(\frac{T}{300}\right)^{-2.15}, \cdots \cdots \cdots \cdots(9)
$$

where $\mu_{n}(300)$ is the carrier mobility at $300 \mathrm{~K}$.

The model is implemented in MATLAB/Simulink as shown in Fig. 4. The junction voltage $V_{d}$ and the total diode voltage $V_{A K}$ are calculated as follows:

$$
\begin{aligned}
\frac{d V_{d}}{d t} & =\frac{I_{A}-I_{d}}{C_{j}} \ldots \ldots \ldots \ldots \\
V_{A K} & =V_{d}+V_{B}=V_{d}+I_{A} R_{B}
\end{aligned}
$$

2.2 Inductive Switching Simulation The device models are implemented in a commutation cell to model the inductive switching processes in the inverter ${ }^{(6)(12)}$. Figure 5 shows the commutation cell circuit, which models the switching process for both pairs of devices in each inverter leg. This contains the freewheel diode, whether Si PIN or SiC Schottky, the IGBT, a small snubber and the necessary stray inductances. This is also implemented in Simulink. One complete switching cycle is simulated in this commutation cell. The temperature is assumed to be fixed throughout the short duration of the cycle.

This inductive switching simulation is then used to calculate the instantaneous device power losses, simply using $P_{D}=I_{A} V_{A K}$ and $P_{I}=I_{C} V_{C E}$ for the diode and IGBT respectively. At each point in the look-up table, i.e. for each combination of load current, duty ratio and temperature, the device power dissipation is averaged across the switching cycle to calculate the total conduction and switching losses ${ }^{(6)}$.

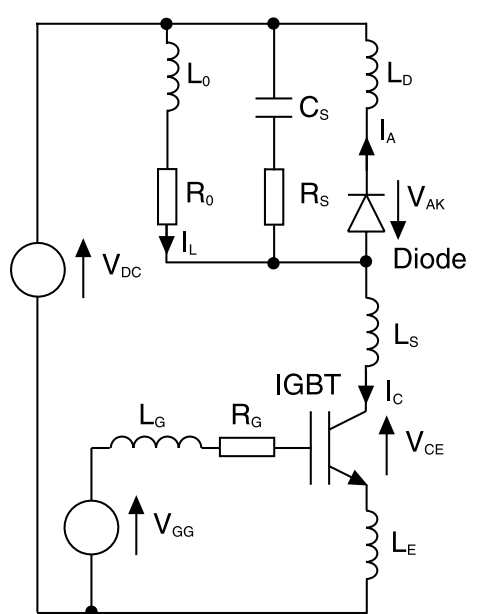

Fig. 5. Chopper cell circuit used to simulate inductive switching. $R_{0}, L_{0}$ : load, $R_{S}, C_{S}$ : snubber, $L_{S}$ : primary stray inductance, $L_{D}$ : diode inductance, $L_{E}$ : emitter Kelvin inductance, $R_{G}, L_{G}$ : gate resistance and inductance

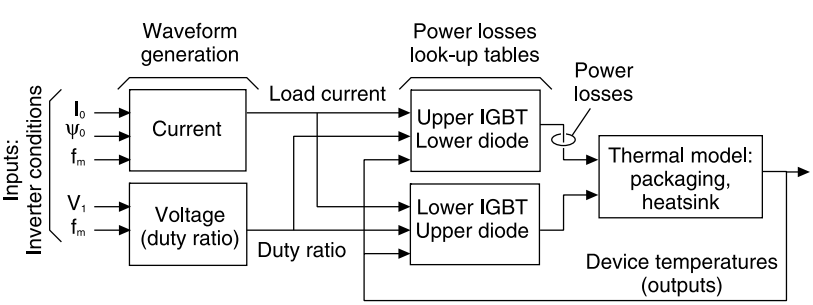

Fig. 6. Outline of the inverter and heatsink model, showing the feedback from the device temperatures to the power losses estimation

2.3 Inverter and Heatsink Model The inverter model outline is given in Fig. 6. The pre-calculated inverter output (motor) electrical conditions ( $I_{0}$ : output current, $V_{1}$ : output voltage, $\varphi_{0}$ : output current phase angle, $f_{m}$ : output modulation frequency) are used to calculate the current and voltage waveforms as the simulation proceeds, which give the load current and duty ratio for the switching cycle of each inverter leg. Only one leg is simulated here, since the legs behave identically except for the $120^{\circ}$ phase difference between legs. The active devices in each leg are selected based on the direction of the load current; therefore the switching conditions of the duty ratio and load current may be calculated. The device temperature is obtained from the heatsink model. The DC supply voltage and switching frequency are assumed to be constant. The switching conditions are then used to access the look-up table of device power losses, with separate losses calculated for all four devices in each inverter leg. The heatsink model, given in Fig. 7, includes the device and packaging transient thermal impedance $\left(Z_{I C}, Z_{D C}\right)$ and the thermal impedance to the heatsink. The capacitances and resistances in $Z_{I C}$ represent the transient impedance, and a similar network is present inside $Z_{D C} . R_{C H}$ is the thermal resistance between the module and heatsink, and $R_{H 1}, R_{H 2}, C_{H 1}$, and $C_{H 2}$ represent the thermal impedance of the heatsink. The baseplate temperature is measured as shown as $T_{M E A S}$. This model calculates the device temperatures from the power losses, also taking account of some cross-coupling of device temperatures. The device thermal impedances are typically 


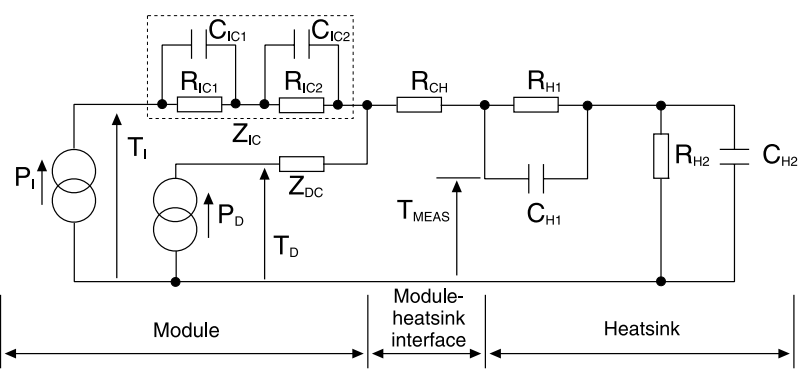

Fig. 7. Device packaging and heatsink model. $P_{I}$ and $P_{D}$ represent the IGBT and diode power dissipations respectively

given in the device datasheet and the heatsink impedances may be estimated from experimental tests, e.g. using known powers from resistive heaters. While the heatsink model is a simple lumped-element thermal network, more detailed twoor three-dimensional models could be used at greater computational expense, or used to generate more accurate lumped element models ${ }^{(5)(8)(15)}$

2.4 Motor Drive Model A variable-speed induction motor drive is used in this part of the model. The motor is assumed to operate in the linear region of the torque-speed characteristic, with variable-voltage variable-frequency control to maintain constant flux below the base speed. Thus the model is simplified, with only the rotor resistance and magnetizing inductance required. These are selected to match the motor size used in the powertrain simulation. Further details of the model are given in Ref. (7).

2.5 Powertrain Model Using a driving cycle to define the vehicle speed with respect to time requires an inverse physical powertrain model to calculate the required powertrain torque. The powertrain model, known as 'WARPSTAR', facilitates this calculation, and is described in more detail in Ref. (16). The powertrain supervisory controller determines the balance of torque between the electric drive and engine to meet the demand, so the control strategy employed largely dictates the torque request on the electric drive. A generic strategy, which maintains the battery state of charge and optimizes the combined efficiency of the system, is suitable for this model.

\section{Results}

3.1 Schottky Diode Model The model was fitted to experimental data from a SiC Schottky diode. This was achieved by varying the principal device structural parameters $\left(L, N_{B}, A, n\right.$, and $\left.\phi_{B n}\right)$ to match the experimental data. Initial estimates for $A, L$ and $N_{B}$ can be made from the current rating, differential on-state resistance, junction capacitance and breakdown voltage given in the datasheet.

Using SiC material data, Fig. 8 shows the comparison between experimental data from Ref. (14), for a $1 \mathrm{~A} / 600 \mathrm{~V}$ device with an area of $0.009 \mathrm{~cm}^{2}$, and simulated on-state curves. The SiC diode model shows close matching to the experimental data.

The SiC Schottky diode model was then simulated in the commutation cell, as in Fig. 5. Nominal switching conditions are chosen to compare the Si PIN diode and SiC Schottky diode. The supply voltage was $200 \mathrm{~V}$, the load current was $200 \mathrm{~A}$ and the temperature was $300 \mathrm{~K}$. The IGBT and

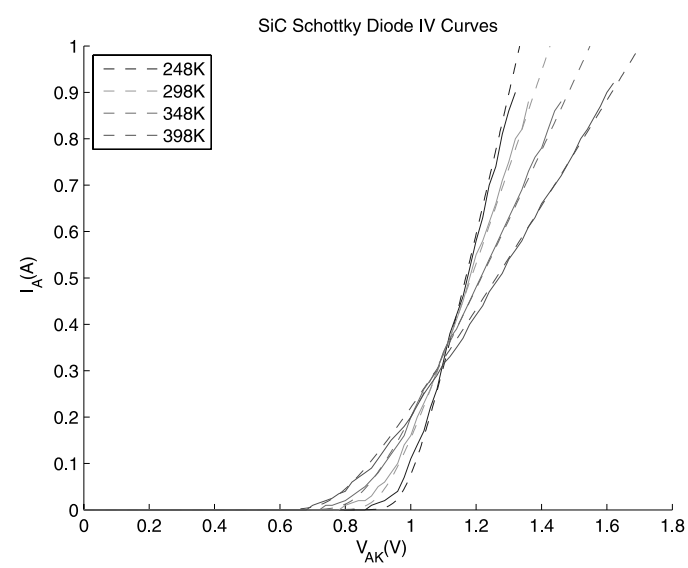

Fig. 8. Comparison of measured (solid) ${ }^{(14)}$ and simulated (dashed) on-state curves for the SiC Schottky diode
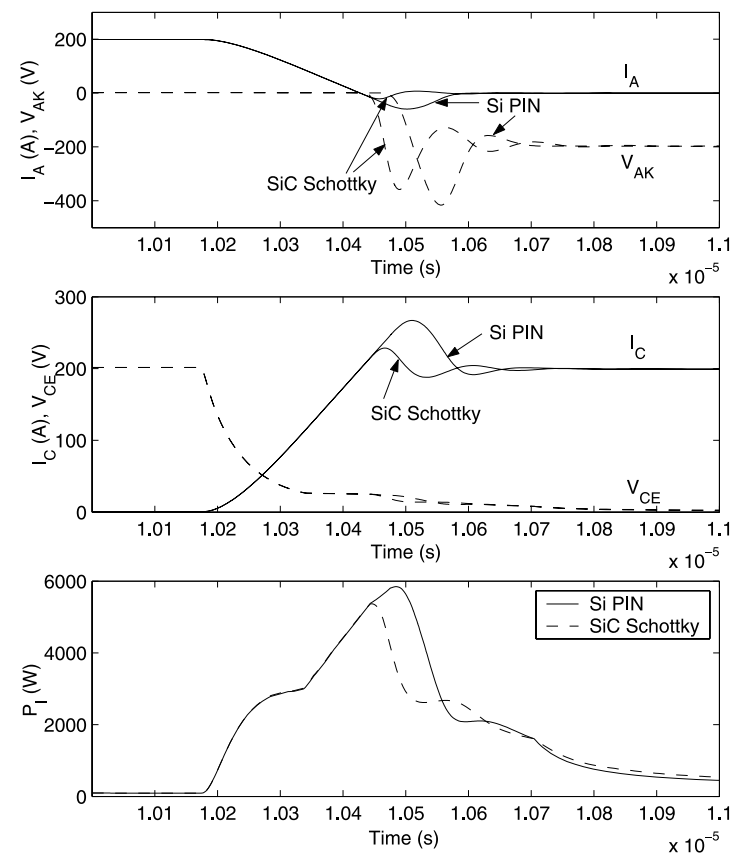

Fig. 9. Switching waveforms for IGBT turn-on/diode turn-off at $300 \mathrm{~K}$. Top: Diode current $I_{A}$, diode voltage $V_{A K}$. Middle: IGBT current $I_{C}$, IGBT collector voltage $V_{C E}$. Bottom: IGBT power dissipation $P_{I}$

PIN diode were rated at 200 A. The area of the SiC Schottky diode was scaled up from that matched in Fig. 8 to achieve a suitable current rating; this could also be viewed as paralleling a suitable number of devices. Figure 9 shows the IGBT turn-on/diode turn-off waveforms for the $\mathrm{SiC}$ Schottky diode, compared with the Si PIN diode. The Schottky diode shows no reverse recovery, although it does show a small oscillation in current due to the interaction of the stray inductance with the diode capacitance. The snubber across the diode had to be increased to $2.7 \mathrm{nF}$ in series with $10 \Omega$ in order to damp these oscillations sufficiently; however this did not significantly increase the diode current overshoot. The power dissipation waveforms in Fig. 9 show the effect of using the SiC Schottky diode on the IGBT turn-on losses. The current overshoot is reduced, with a resulting reduction in switching losses.

Figure 10 compares the on-state characteristics of the SiC Schottky and Si PIN diodes, with two $200 \mathrm{~A}$ devices in 


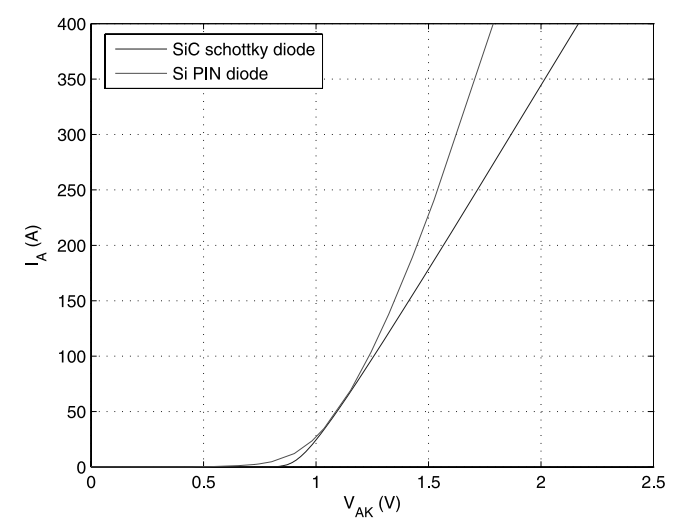

Fig. 10. Comparison of Si PIN diode and SiC Schottky diode on-state characteristics at $300 \mathrm{~K}$ (two $200 \mathrm{~A}$ devices in parallel)
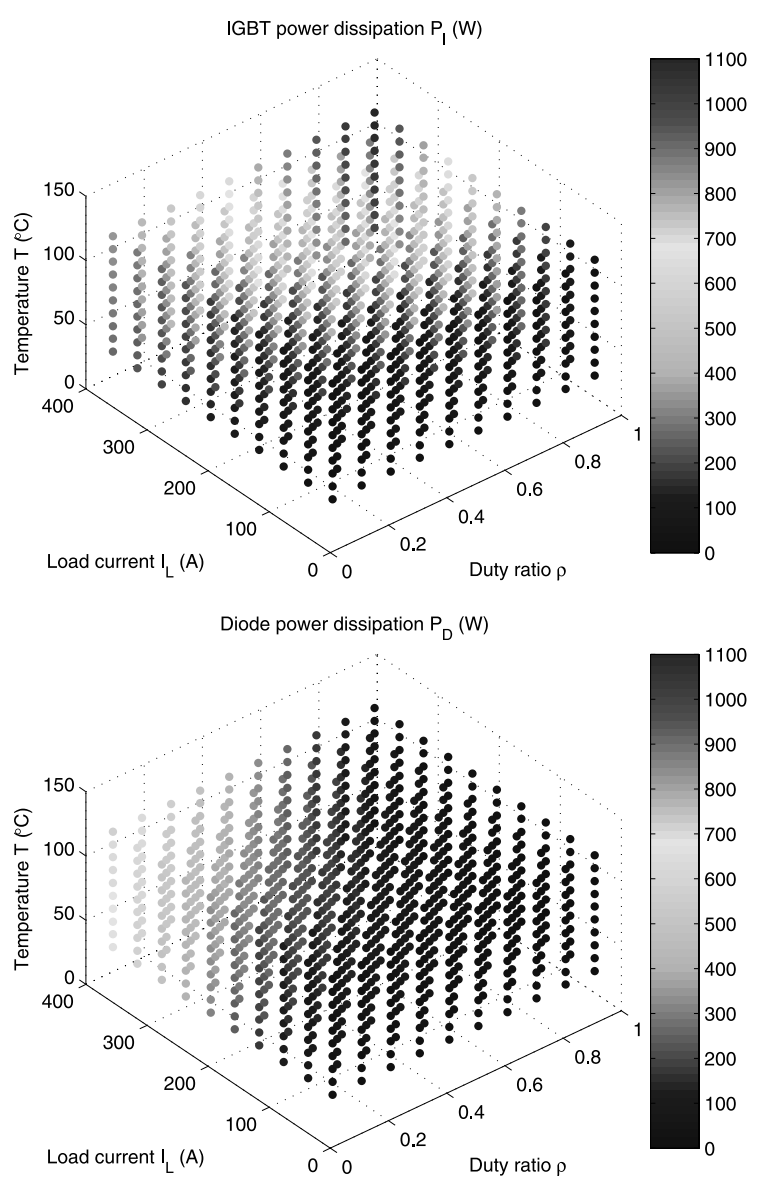

Fig. 11. Look-up tables for the device power dissipation, with the Si PIN diode used at $5 \mathrm{kHz}$. The colour scale indicates the power dissipation in watts

parallel for each curve. The chip area of the SiC Schottky diode is more than double that of the Si PIN diode. The consequence of using a minority carrier device is the increased resistance at higher current densities and temperatures; in addition the greater bandgap of $\mathrm{SiC}$ gives a larger "knee" voltage before conduction starts than a Si Schottky diode. Although the on-state voltages of the diodes are similar below about 150 A (75 A per chip), the SiC Schottky diode exhibits a greater on-state voltage above this. The on-state voltage of the SiC Schottky diode could be reduced by increasing the device area, but this would increase the cost and reduce the
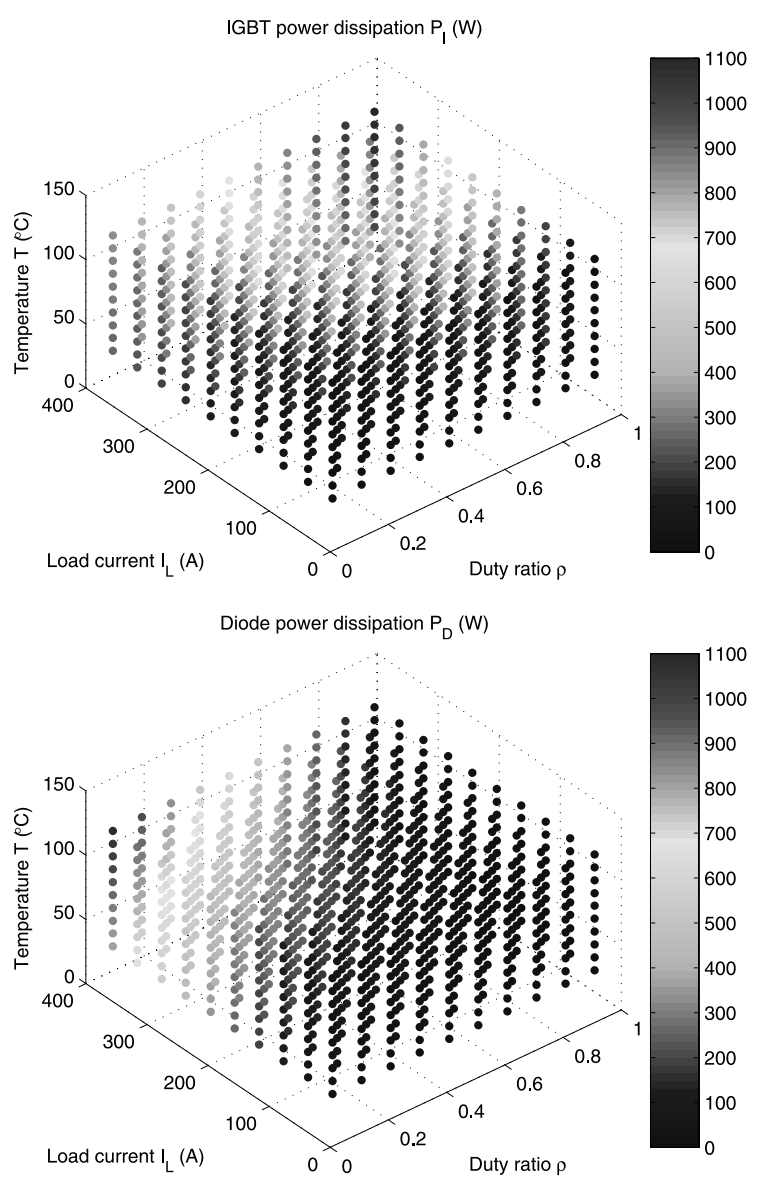

Fig. 12. Look-up tables for the device power dissipation, with the SiC Schottky diode used at $5 \mathrm{kHz}$. The colour scale indicates the power dissipation in watts

effective utilization of the device.

3.2 Simulation of Device Losses The Si devices chosen are based on an IGBT/PIN diode pair previously modelled using the Si device models. The resulting look-up table of average device power losses using Si PIN diodes is shown in Fig. 11. The switching frequency was $5 \mathrm{kHz}$ and the DC supply voltage was $240 \mathrm{~V}$ to match the nominal battery voltage in the powertrain simulation. The device losses increase with current and temperature, as expected. Since the switching frequency is relatively low, the balance of the losses between the IGBT and diode is strongly affected by the duty ratio.

Figure 12 shows the corresponding look-up table of power losses using the SiC Schottky diode. Since the switching frequency is relatively low, and the on-state losses of the SiC Schottky diode are higher than that of the Si PIN diode, the losses are greater than those given in Fig. 11. This is shown in the lower plot of each figure (diode losses).

However, at a switching frequency of $25 \mathrm{kHz}$, the total device (IGBT + diode) losses are similar, Fig. 13; indeed there is little difference between the two plots. This illustrates the improved performance of SiC Schottky diode at high switching frequencies, as expected from the reduced switching losses, the overall losses become comparable. At higher switching frequencies still, the total losses using the SiC Schottky diode would be lower than using the Si PIN diode. 


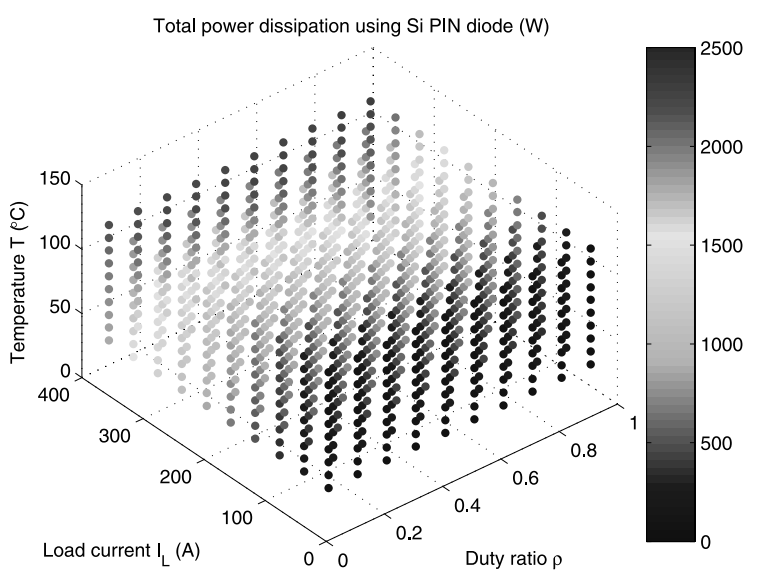

Total power dissipation using SiC Schottky diode (W)

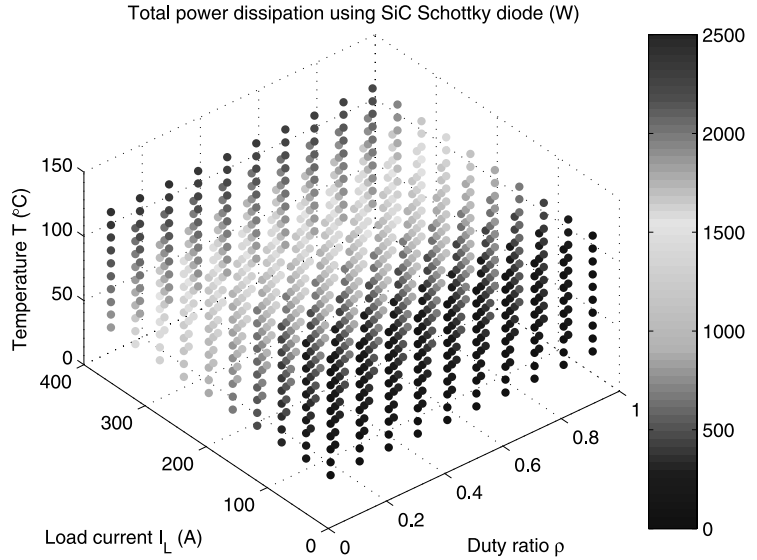

Fig. 13. Look-up tables for the total device power dissipation at a switching frequency of $25 \mathrm{kHz}$. Upper: $\mathrm{Si}$ PIN diode, lower: SiC Schottky diode. The colour scale indicates the power dissipation in watts

3.3 Powertrain, Motor Drive and Inverter Simulation The candidate hybrid vehicle used in this paper is a D-segment vehicle, examples of which are the Ford Mondeo and Volkswagen Passat. A full parallel hybrid system is chosen to illustrate the simulation framework. A generic chassis mass of 1.5 tonnes and generic aerodynamic properties of 0.34 for drag coefficient and $2 \mathrm{~m}^{2}$ for frontal area are used. The full hybrid has a $988 \mathrm{cc}$ petrol engine, with a $60 \mathrm{~kW}$ induction machine combined motor/generator. It has a 5-speed manual transmission, with gear selection by a medium conservative driver model. It should be emphasised that this is an example, and any hybrid electric powertrain could be used here, provided it is accounted for correctly in the powertrain model. The driving cycles used to provide the vehicle speed demand are the Artemis urban and motorway cycles, combined into one cycle, and the NEDC.

The inverter is assumed to be a three-phase voltage source inverter, with a constant DC link voltage of $240 \mathrm{~V}$ and a switching frequency of $5 \mathrm{kHz}$, using space-vector modulation to generate the voltage waveforms. The power losses are calculated for one leg as discussed in section 2.3.

Figure 14 shows the relationship between the vehicle speed, motor speed, motor torque, inverter output voltage and load current for the vehicle subjected to the combined Artemis cycle (consisting of an urban cycle followed by a motorway cycle). The load current clearly corresponds
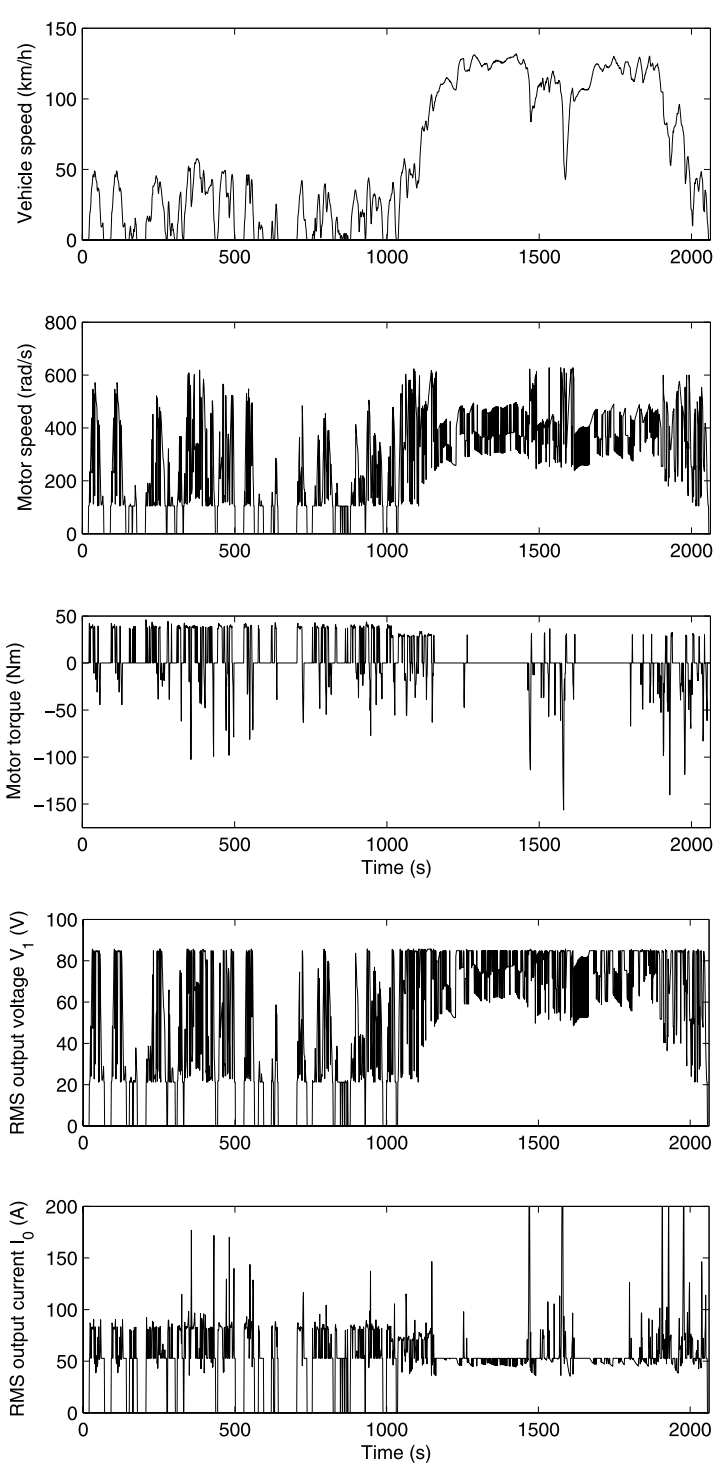

Fig. 14. Speeds, torque, motor voltage and current for the Artemis driving cycle. The urban section is followed by the motorway section at approximately $1000 \mathrm{~s}$

to the motor torque. The motor speed is not directly to the vehicle speed, due to the gear changes, but directly affects the inverter voltage. Using such a load cycle automatically includes common test conditions such as halfload and full-load operation, since these are encountered during the constant-speed highway section and acceleration/deceleration sections of the cycle. The output current $I_{0}$ is not always zero for zero motor torque, because of the need to maintain flux in the machine.

Figure 15 shows the device temperatures, using both the Si PIN and SiC Schottky diodes, at a switching frequency of $5 \mathrm{kHz}$. The temperatures clearly depend on the vehicle torque, with peaks in temperature corresponding to the vehicle acceleration and magnitude of the torque. The temperatures show greater peaks and more severe cycling during the urban section, which would adversely impact the device reliability. The maximum temperature rise occurs during sharp braking, due to regeneration of power to the battery. This is somewhat unexpected, but is highly dependent on the powertrain management strategy, which would dictate the balance 

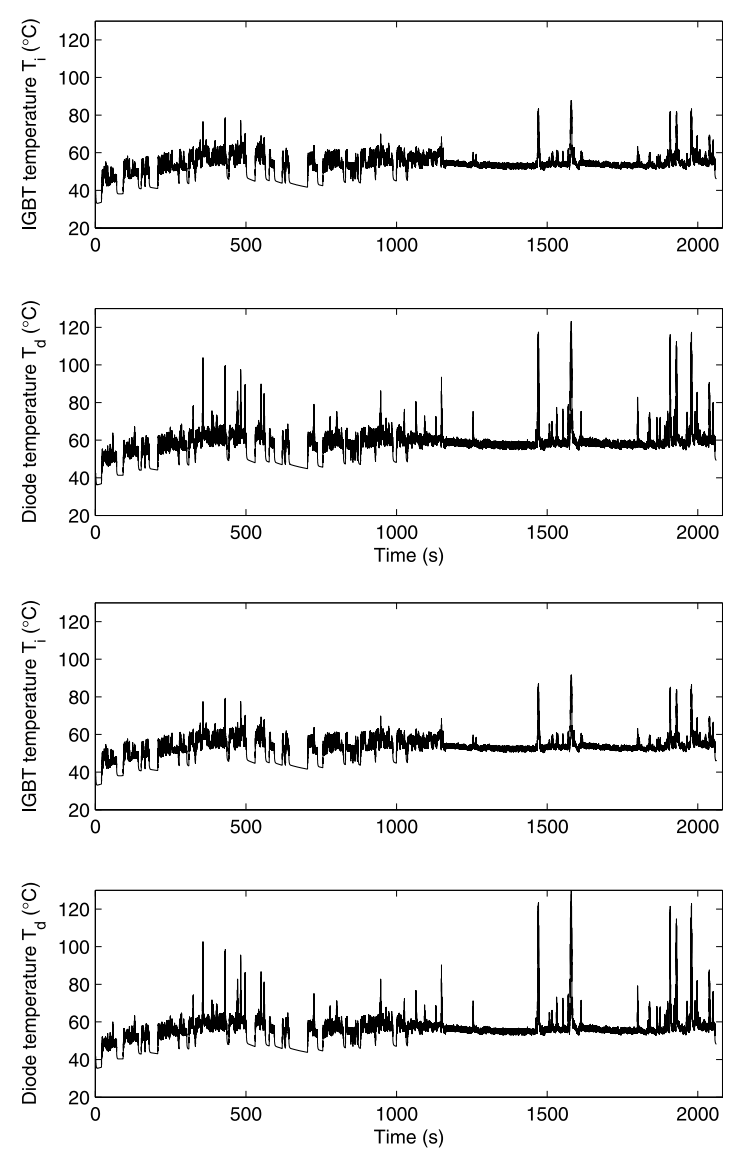

Fig. 15. IGBT and diode temperatures for the Artemis driving cycle. Upper: Si PIN, lower: SiC Schottky

of regenerative and conventional friction braking. There is little difference between the device temperatures simulated using the Si PIN diode and those using the SiC Schottky diode. This is because of the strong dependence on the onstate losses at the switching frequency used, and the maximum device current being approximately 200 A (Fig. 10). It should also be noted that the temperatures depend on the packaging and heatsink design, and the accuracy of the simulated temperatures depend on the thermal model.

Figure 16 shows the relationship between the vehicle speed, motor speed and motor torque for the vehicle subjected to the NEDC cycle. The acceleration is smaller than for the Artemis cycle, with a correspondingly reduced load current and device temperature. Figure 17 shows the device temperatures, using both the Si PIN and SiC Schottky diodes, at a switching frequency of $5 \mathrm{kHz}$.

Both driving cycles show that the diode temperature is greater than the IGBT temperature. This is because the thermal resistance between the diode chip and the heatsink is greater than for the IGBT, due to the smaller area of the diode chip. Additionally, the SiC Schottky diode chip is larger than the Si PIN diode chip, and the thermal conductivity of $\mathrm{SiC}$ is greater. Therefore the smaller thermal resistance of the SiC diode compared with the Si PIN diode counteracts the larger power dissipation expected from Fig. 12.

Generation of the look-up tables using the device models switching at $5 \mathrm{kHz}$ took $259 \mathrm{~s}$ and $97 \mathrm{~s}$ on a $3 \mathrm{GHz}$ Pentium IV PC for the Si PIN and SiC Schottky diodes respectively.
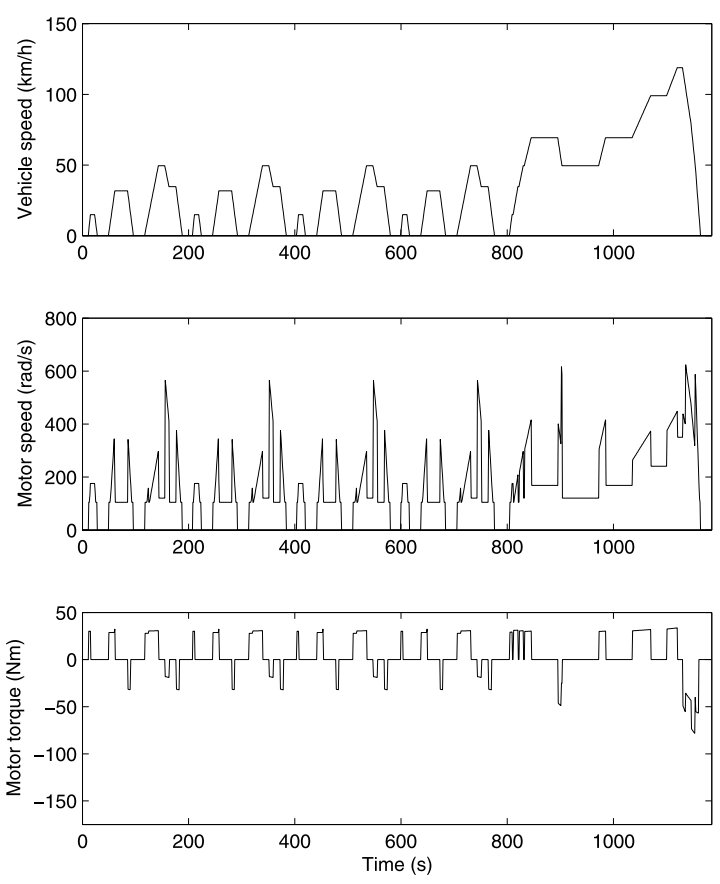

Fig. 16. Speeds and torque for the NEDC driving cycle
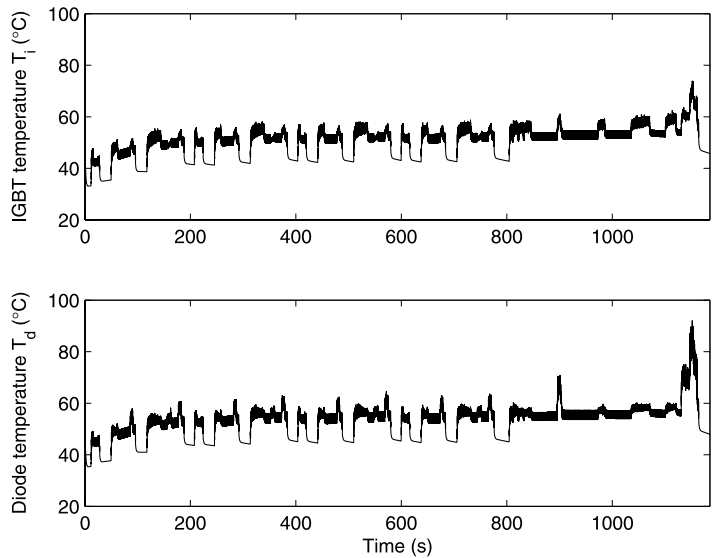

Fig. 17. IGBT and diode temperatures for the NEDC driving cycle, using the SiC Schottky diode

The simulation with the PIN diode takes longer because of the need to model the stored charge, which is more complex than the Schottky diode model. The inverter simulations took $370 \mathrm{~s}$ and $167 \mathrm{~s}$ for the combined Artemis and NEDC cycles respectively for the Si PIN diode, and $376 \mathrm{~s}$ and $169 \mathrm{~s}$ for the SiC Schottky diode. The Artemis cycle takes longer than the NEDC cycle simply because it is longer. This results in speed-up factors of at least 3.3 times faster than real time (2082 s load cycle simulated in $629 \mathrm{~s}$ for the worst case of the Si PIN diode look-up table generation and Artemis cycle). To compare this with having to simulate every single switching cycle, if every switching cycle takes ten seconds to simulate, and the switching frequency is $5 \mathrm{kHz}$, the Artemis load cycle of $2082 \mathrm{~s}$ would take $1.04 \times 10^{8} \mathrm{~s}$ to simulate, i.e. 3.3 years. Therefore it is clear that using the look-up table method for inverter simulation is the only feasible option.

\section{Discussion}

The results presented show that the case for using new 
material devices such as SiC Schottky diodes is not necessarily clear-cut. The example shown here achieves a similar performance to an already well-optimized Si PIN diode, at the expense of using a diode chip area more than double that of the Si PIN diode. A larger area still would improve the performance beyond that of the Si PIN diode, but would be more expensive and more difficult to package. The potential drawback of high on-state losses of SiC Schottky diodes has not been widely discussed-although with some exceptions, e.g. in Ref. (17)—since it is assumed that such diodes operate at high switching frequencies. This result goes against many currently-held assumptions of the benefits of SiC devices, and would also extend to other motor drive inverter systems. However it is not surprising once the relatively low switching frequency used here $(5 \mathrm{kHz})$ is taken into account.

However, the ability of $\mathrm{SiC}$ to operate at higher temperatures would not restrict the diode temperature to the same extent, allowing smaller diode chip sizes to be used. This could, however, increase the temperature of an adjacent IGBT, so this thermal coupling must be taken into consideration. It would also require reliable packaging capable of withstanding the severe thermal cycling resulting from increased device temperatures. The thermal cycling experienced by the devices is already severe, as shown in Figs. 15 and 17. The estimation of device reliability is one area in which this simulation could be used in the design of the devices and inverter $^{(6)}$.

At higher switching frequencies, the total losses of the inverter using the Si PIN diode approach those of the SiC Schottky diode, as shown by the losses at $25 \mathrm{kHz}$, Figs. 13. This shows the expected advantage of the SiC Schottky diode taking effect: as it is a majority carrier device there is no reverse recovery current, so the switching losses are greatly reduced in the IGBT and eliminated in the diode. The advantages of near-zero switching losses and higher operating temperatures of SiC Schottky diodes are expected to be fully realizable with the use of SiC MOSFET or JFET devices, which, because of the near-zero switching losses, would allow the switching frequencies to be greatly increased. The cost of increasing the switching frequency, in terms of device temperatures, can quickly be evaluated using the fast inverter simulation, allowing different design options to be investigated thoroughly.

At this stage the inverter simulation can be used as an exploratory tool to determine the dominant trends in inverter design for hybrid-electric vehicles. It can also be applied to any motor drive system or inverter application provided the inverter output conditions can be calculated. Past results in Ref. (7) indicate that the inverter simulation method is valid, and further work is in progress to fully validate the approach. The use of the simple lumped-element thermal heatsink model also limits the accuracy currently achievable. Further work is in progress to improve the thermal model accordingly.

\section{Conclusions}

The results illustrate the integrated simulation framework developed for inverter design in hybrid vehicle powertrains. It is a powerful tool, with the advantage that all stages of the simulation are within MATLAB/Simulink. It offers for the first time a method of estimating rapidly the temperatures of the power devices in the inverter throughout the whole driving cycle. The accuracy of power losses and detail of device temperature is maintained throughout the inverter simulation. The total simulation time of approximately 10 minutes, from the device and inverter specification to the complete temperature profile, offers great potential in inverter optimization since many designs can be evaluated practically. It has also shown that the use of SiC Schottky diodes in conjunction with Si IGBTs in hybrid vehicle systems does not necessarily show the improvements in performance expected.

(Manuscript received May 11, 2007,

revised Nov. 6, 2007)

\section{References}

( 1 ) A.R. Hefner: "A dynamic electro-thermal model for the IGBT", IEEE Trans. Industry Applications, Vol.30, No.2, pp.394-405 (1994-3)

( 2 ) T. Kojima, Y. Yamada, M. Ciappa, M. Chiavarini, and W. Fichtner: "A novel eletcro-thermal simulation approach of power IGBT modules for automotive traction applications", In ISPSD Conf. Rec., Kitakyushu (2004)

( 3 ) A.D. Rajapakse, A.M. Gole, and P.L. Wilson: "Electromagnetic transient simulation models for accurate representation of switching losses and thermal performance in power electronic systems", IEEE Trans. Power Delivery, Vol.20, No.1, pp.319-327 (2005-1)

( 4 ) J. Reichl, J.-S. Lai, A. Hefner, T. McNutt, and D. Berning: "Inverter dynamic electro-thermal modeling and simulation with experimental verification", In PESC Conf. Rec., Recife (2005-6)

( 5 ) Y.C. Gerstenmaier, A. Castellazzi, and G.K.M. Wachutka: "Electrothermal simulation of multichip-module with novel transient thermal model and timedependent boundary conditions", IEEE Trans. Power Electronics, Vol.21, No.1, pp.45-55 (2006-1)

( 6 ) A.T. Bryant, P.A. Mawby, P.R. Palmer, E. Santi, and J.L. Hudgins: "Exploration of power device reliability using compact thermal models and fast electro-thermal simulation", In IAS Conf. Rec., Tampa (2006-10)

( 7 ) A.T. Bryant, N-A. Parker-Allotey, and P.R. Palmer: "The use of condition maps in the design and testing of power electronic circuits and devices", IEEE Trans. Industry Applications, Vol.43, No.4, pp.874-883 (2007-7)

( 8 ) Z. Zhou, M.S. Khanniche, P. Igic, S.T. Kong, M. Towers, and P.A. Mawby: "A fast power loss calculation method for long real time thermal simulation of IGBT modules for a three-phase inverter system", Int. J. Numerical Modelling: Electronic Networks, Devices and Fields, Vol.19, pp.33-46 (2006) Also in EPE Conf. Rec., Dresden (2005-9)

( 9 ) P.R. Palmer, E. Santi, J.L. Hudgins, X. Kang, J.C. Joyce, and P.Y. Eng: "Circuit simulator models for the diode and IGBT with full temperature dependent features", IEEE Trans. Power Electronics, Vol.18, No.5, pp.1220-1229 (2003-9)

(10) A.T. Bryant, L. Lu, E. Santi, P.R. Palmer, and J.L. Hudgins: "Physical modeling of fast p-i-n diodes with carrier lifetime zoning, part I: device model", IEEE Trans. Power Electronics, Vol.23, No.1, pp.189-197 (2008-1)

(11) X. Kang, A. Caiafa, E. Santi, J.L. Hudgins, and P.R. Palmer: "Characterization and modeling of high-voltage field-stop IGBTs", IEEE Trans. Industry Applications, Vol.39, No.4, pp.922-928 (2003-7/8)

(12) A.T. Bryant, P.R. Palmer, E. Santi, and J.L. Hudgins: "Simulation and optimisation of diode and IGBT interaction in a chopper cell using MATLAB and Simulink", IEEE Trans. Industry Applications, Vol.43, No.4, pp.902-910 (2007-7)

(13) S.M. Sze: "Physics of Semiconductor Devices", John Wiley and Sons, second edition (1981)

(14) T.R. McNutt, A.R. Hefner, H.A. Mantooth, J. Duliere, D.W. Berning, and R. Singh: "Silicon carbide PIN and merged PIN schottky power diode models implemented in the Saber circuit simulator", IEEE Trans. Power Electronics, Vol.19, No.3, pp.573-581 (2004-5)

(15) M. Ciappa, W. Fichtner, T. Kojima, Y. Yamada, and Y. Nishibe: "Extraction of accurate thermal compact models for fast electro-thermal simulation of IGBT modules in hybrid electric vehicles", Microelectronics Reliability, Vol.45, pp.1694-1699 (2005)

(16) A. Walker, A. McGordon, G. Hannis, A. Picarelli, J. Breddy, S. Carter, A. Vinsome, P. Jennings, M. Dempsey, and M. Willows: "A novel structure for comprehensive HEV powertrain modelling", In VPP Conf. Rec., Windsor (2006-9) 
(17) A. Elasser, M.H. Kheraluwala, M. Ghezzo, R.L. Steigerwald, N.A. Evers, J. Kretchmer, and T.P. Chow: "A comparative evaluation of new silicon carbide diodes and state-of-the-art silicon diodes for power electronic applications", IEEE Trans. Industry Applications, Vol.39, No.4, pp.915-921 (2003-7)

Angus Bryant (Non-member) received the MEng degree in electri-

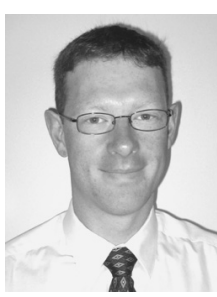
cal and information sciences and the $\mathrm{PhD}$ degree in power electronics from Queens' College, Cambridge University, Cambridge, UK, in 2001 and 2005, respectively. He was a Research Fellow at the School of Engineering, University of Warwick, UK, where he worked on power device modeling in projects with Toyota Motor Corporation and GE Aviation. He became an Assistant Professor at the University of Warwick in 2007. His main interests are semiconductor device modeling, simulation and characterization of power electronic systems, and optimization and testing of power electronic systems under realistic loading conditions.

Graham Roberts (Non-member) was born in Gravesend, England in

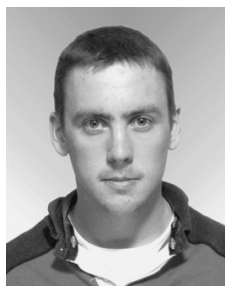
1984. He received the MEng degree in electronic engineering with first class honours from Warwick University, UK, in 2006, where he is currently studying for the $\mathrm{PhD}$ degree in the Electrical and Electronics division of the School of Engineering. His research to date has been on the temperature dependent modelling of $\mathrm{SiC}$ power devices and improvement of channel mobility in SiC MOSFETs.

Alan Walker (Non-member) gained an $\mathrm{MSc}$ and $\mathrm{PhD}$ from Impe-

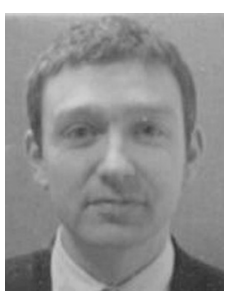
rial College, London, UK, in Hybrid Vehicle Electric Drives. He has held automotive research positions with Haldex AB, PARD, and now with AVL Powertrain UK. His current research activities include hybrid vehicles, electric traction systems, simulation and control.

Philip Mawby

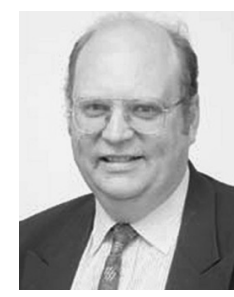

(Non-member) graduated from the University of Leeds, UK, and obtained his $\mathrm{PhD}$ from the same institution in 1986, where he studied GaAs/AlGaAs heterojunction bipolar transistors for high power radio frequency applications in conjunction with coworkers at the GEC Hirst Research Centre in Wembley, UK. From 1986-2005 he was at the University of Wales, Swansea, where has built an international reputation in the area of power electronics and power device research, and held the Royal Academy of Engineering Research Chair, sponsored by the Welsh Development Agency. His main interests are materials for new power devices, modelling of power devices and circuits, power integrated circuits, as well as their applications. He has also worked extensively on development of device simulation algorithms, as well as optoelectronic and quantum based device structures. Since 2005 has held the Chair of Power Electronics at the University of Warwick, and is Director of the Warwick Institute for Sustainable Energy and Resources. He is on many international conference committees, including ISPSD, EPE, BCTM and ESSDERC. He is Chartered Engineer, a Fellow of the IET, and a Fellow of the Institute Physics as well as a Senior Member of the IEEE. He has published over 70 journal papers as well as over 100 conference papers, and is a distinguished lecturer for the IEEE Electron Devices Society.
Takashi Ueta (Non-member) was born in Fukuoka, Japan in 1966.

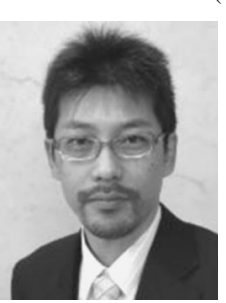
He received a B.Eng. degree in 1990 and an M.Eng. degree in 1992 both in electronic engineering from Fukuoka University in Japan. In 1992 he joined Toyota Motor Corporation in Aichi, Japan. In 1992 he was involved in the in-house semiconductor project and in charge of analyses technologies, simulation technologies and $\mathrm{Si}$ device technologies such as Power MOSFET, IGBT. He is now a group manager of the group in charge of $\mathrm{CAD} / \mathrm{CIM}$ technologies.

Toshifumi Nishijima (Non-member) was born in Aichi, Japan in

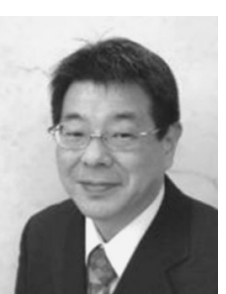
1964. He received a B.Eng. degree in electrical engineering from Meijo University, Japan, in 1986. In 1990 he joined Toyota Motor Corporation in Aichi, Japan. He was involved in the in-house semiconductor project and in charge of developing LSI technologies and $\mathrm{CAD} / \mathrm{CIM}$ technologies. He is now a Group Manager of the group in charge of LSI for multimedia system.

Kimimori Hamada (Member) was born in Osaka, Japan in 1959.

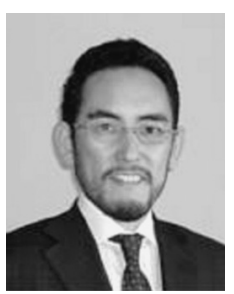
He received a B.Eng. degree in 1982 and an M.Eng. degree in 1985 both in electrical engineering from Osaka Prefecture University in Japan. In 1985 he joined Toyota Motor Corporation in Aichi, Japan. In 1987 he was involved in the in-house semiconductor project and in charge of developing process technologies and $\mathrm{Si}$ device technologies such as Power MOSFET, IGBT, and BiCDMOS. He is now a general manager of the department in charge of developing novel devices, and CAD/CIM technologies. He is a member of JSAE and IEEJ. 